\title{
Asymmetric Organocatalysis with Chiral Covalent Organic Frameworks
}

\author{
Song-Chen $\mathrm{Yu}^{\mathrm{a}, \mathrm{b}}$ (1) \\ Liang Cheng*a,b,c (i) \\ Li Liu*a,b \\ a Beijing National Laboratory for Molecular Sciences (BNLMS), CAS Key Laboratory of \\ Molecular Recognition and Function, CAS Research/Education Center for Excellence \\ in Molecular Sciences, Institute of Chemistry, Chinese Academy of Sciences, Beijing \\ 100190, China \\ b University of Chinese Academy of Sciences, Beijing 100049, China \\ 'Hangzhou Institute for Advanced Study, University of Chinese Academy of Sciences, \\ Chinese Academy of Sciences, Hangzhou 310024, China \\ chengl@iccas.ac.cn; lliu@iccas.ac.cn \\ Dedicated to Prof. emeritus Dong Wang on the occasion of his 80th birthday.
}

Received: 14.12 .2020

Accepted after revision: 24.02.2021

DOI: 10.1055/a-1400-5581; Art ID: om-20-0043sr

License terms: (C)

(c) 2021. The Author(s). This is an open access article published by Thieme under the terms of the Creative Commons Attribution-NonDerivative-NonCommercial License, permitting copying and reproduction so long as the original work is given appropriate credit. Contents may not be used for commercial purposes, or adapted, remixed transformed or built upon. (https://creativecommons.org/licenses/by-nc-nd/4.0/)

Abstract Inspired by Mother Nature, the use of chiral covalent organic frameworks as heterogeneous asymmetric organocatalysts has arisen over the last decade as a new method in enantioselective synthesis. In this Short Review, sophisticated design of these polymeric materials and their application in asymmetric organocatalysis will be discussed.

Key words chiral covalent organic frameworks, post-modification, asymmetric organocatalysis, enantioselective synthesis

\section{Introduction}

Chirality is a universal property of asymmetry that is essential in most branches of science. ${ }^{1,2}$ Most biological macromolecules like nucleic acids, proteins, and saccharides are presented in only one chiral form. For example, DNA is composed with four D-deoxyriboses while around 20 L-amino acids make up thousands of different proteins. Different enantiomers of exogenous small organic molecules may bind inversely (or even absolutely not) to their target receptors. It is reasonable that one of the enantiomers exhibits preferred properties while the other(s) may cause serious and/or undesired side effects, or occasionally another effects. $^{3}$ Therefore, the design, preparation, and separation of designated enantiomer have a special attraction to chemists, especially in pharmaceutical industry, where chiral drugs predominate.

Among the vast majority of methodologies for the generation of enantiopure compounds, asymmetric cataly-

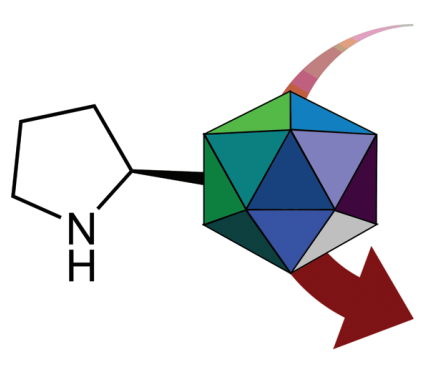

Achiral substrate(s)

Chiral COF

Chiral product(s)

$\mathrm{sis}^{4-6}$ is envisioned as an ideal pathway in which a small amount of a well-designed chiral catalyst, as low as ppm quantity, is capable of transforming achiral starting materials into enantiomeric molecules stereoselectively. The last two decades has witnessed the blossom of asymmetric catalysis prompted by metal-chiral ligand complexes and organocatalysts, which have found wide application from laboratory synthesis of mini-gram scale to industrial manufacture of tons of chemicals. At the same time, their immobilization onto inorganic or organic supports has received considerable attention. $^{7}$ These supported versions of active metal- $/$ organocatalysts exhibited several distinct advantages in terms of their practical use and easy-to-recovery compared with their homogeneous analogues. However, considering the unequal microenvironments of each active site, sometimes inferior asymmetric inductions were observed.

The chemistry behind covalent organic frameworks (COFs), a unique class of crystalline organic polymers with perpetual porosity and extremely well-organized structures, has aroused great interest among chemists in the last decade from diverse fields. ${ }^{8-17}$ With the continuous discovery of new COFs, their applications have significantly expanded from gas adsorption and separation to energy-storage devices, optoelectronics, sensing to drug delivery, and catalysis. ${ }^{18-22}$ Instead of preparing chiral polymers containing several repeating catalytic units, the use of organized and chiral frameworks in enantioselective catalysis is beginning to be investigated. ${ }^{23-31}$ These materials, either with post-modified chiral motifs or inherent asymmetric backbones, fulfilled asymmetric organocatalysis in a confined microenvironment that mimics polymeric enzymes or ribozymes and thus may contribute to the efficiency and selectivity improvement during the catalytic process. Besides, they also possess the advantages of polymeric catalysts in terms of stability in aqueous solutions, strong acids, and bases, which make them a better catalyst than natural enzymes. In this Short Review, this emerging field will be introduced. ${ }^{32}$ 


\section{Biosketches}
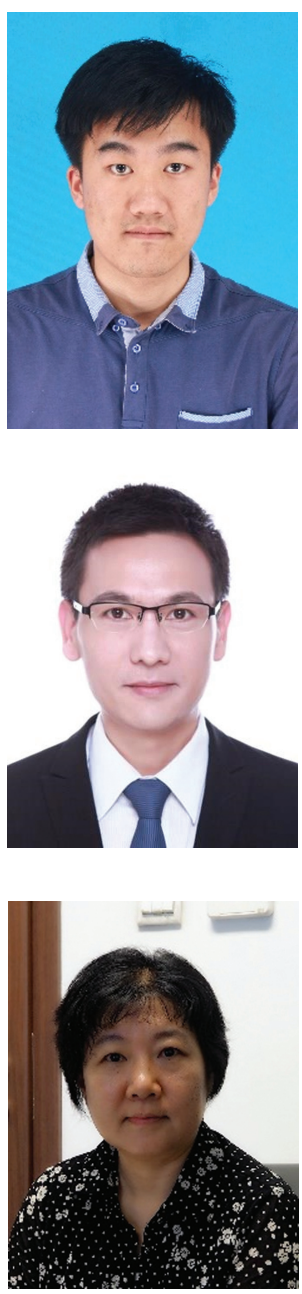
Academy of Sciences. She con-
Sciences. In 2018, he joined Dr. Li Liu's research group at Institute of Chemistry, Chinese Academy of Sciences to pursue a $\mathrm{PhD}$ degree. He focuses his research interest on synthesis and catalytic application of chiral covalent organic frameworks.
Prof. Liu received her PhD from Institute of Chemistry, Chinese Academy of Sciences in 1994. Then she spent 5 years as a lecturer at Graduate School of Chinese

Prof. Cheng received his B.Sc degree from Beijing Normal University in 2004, and PhD from Institute of Chemistry, Chinese Academy of Sciences in 2009. After 2 years in pharmaceutical industry, he joined the laboratory of Prof. Ronald Breslow as a postdoctoral research

ducted postdoctoral research at the Department of Organic Chemistry, University of Ghent, Belgium (1999-2001) and University of Washington at Seattle (2002-2003). From 2003 up to now, she has served as an scientist at the Department of Chemreturned to Institute of Chemistry, Chinese Academy of Sciences in 2015 as an Associate Professor. His research focuses on the development of biorthogonal transformations and chemical modulation of biomacro- molecules. He has been awarded the Young Elite Scientist Sponsorship Program by China Association for Science and Technology, the Excellent Young Scientists Fund of Natural Science Foundation of China, and the Thieme Chemistry Journals Award.
Most COFs reported in literature were achiral, meaning that they are superimposable with their mirror images. There are three types of synthesis methods for the construction of chiral COFs: the chirality-induced postmodification of achiral COF (Figure 1, path a), construction with chiral monomers (path b), ${ }^{33}$ and the use of achiral monomers under asymmetric conditions (path c). ${ }^{34}$ We have chosen not to distinguish between these chirality induction pathways in the preparation of chiral COFs and the influence of post-modification onto the structure of COFs. Instead, a conceptual style will be adopted, focusing on the diverse strategy and modifying approaches that have been proposed and utilized in asymmetric catalysis with chiral COFs. Hopefully this Short Review may inspire more synthetic chemists and materials scientists to devote themselves to this fast-developing field and create more chiral COF materials with interesting properties.
Associate Professor and Professor at Institute of Chemistry, Chinese Academy of Sciences. Her research interest focuses on the development of efficient organic reactions in aqueous media.

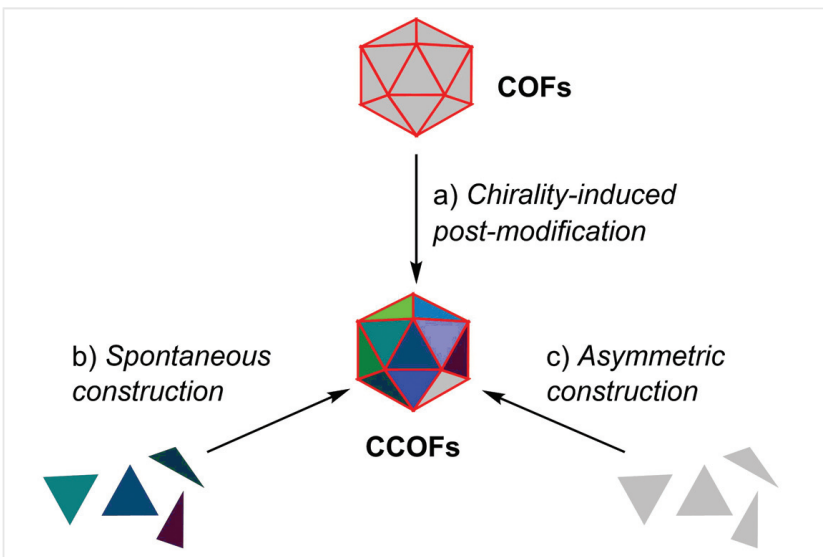

Chiral monomers

Figure 1 Three pathways for the construction of chiral COFs. 


\section{Asymmetric Organocatalysis with Chiral COFs}

Enamine catalysis has been proven as a generic activation mode of carbonyl-containing compounds from its seminal application in the Mannich addition in 2000. ${ }^{35,36}$ Since then, a tremendous amount of efforts has been devoted towards the identification of new types of chiral enamine catalysts. The general mechanism of the typical secondary amine-containing catalyst was that they rapidly interact with an aldehyde/ketone substrate to form an enamine intermediate, which functions as an active nucleophile to engage with another electrophile to generate the adduct (Figure 2). Chiral pyrrolidine is regarded as one of the best enamine catalysts. The simplest and natural L-proline and its derivatives have shown promising efficiency and enantioselectivity in a variety of transformations like the Aldol addition, Michael addition, Mannich addition, amination, etc. ${ }^{37-40}$ The 2-substituted group either functions as a steric hindrance substituent that controls the addition direction (as shown in Figure 2), or forms hydrogen bonding with the electrophiles to fulfill the dual-activation. Generally, a larger substituent always gives a better stereoselectivity. However, sometimes simply increasing the steric group would not benefit the efficiency.

The Michael addition of aldehydes/ketones to nitroalkenes is one of the most-studied $\mathrm{C}-\mathrm{C}$ bond formation reactions, which was regarded as a useful method to obtain $\gamma$-nitro carbonyls. ${ }^{41}$ A diverse range of enantioselective organocatalysts based on pyrrolidines has been developed for this transformation. Luo et al. applied click chemistry into the construction of triazole-based pyrrolidines like $\mathbf{1}$ and evaluated their capability in asymmetric Michael addition of ketones to nitroolefins. ${ }^{42}$ Good to excellent efficiency and stereoselectivities were observed (yields $\leq$ $100 \%$, dr $\leq 99 / 1$, e.e. $\leq 96 \%$ ). The planar triazole ring was proposed to be an excellent space-shielding group compared with traditional bulky substituents. However, the same molecule showed a moderate efficacy in the reaction with aldehyde $\mathbf{2}$ (dr 60/40, e.e. 49\%, Figure 3a, b), probably because the generated enamine was too flexible in the

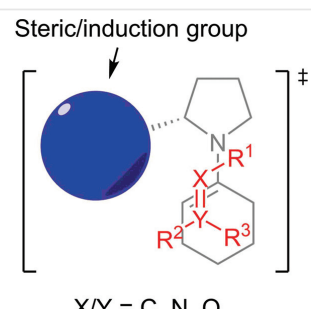

Figure 2 General activation model of secondary amines. (R)-2-Substituted pyrrolidine and cyclohexanone were used for demonstration. The blue cycle represents steric hindrance groups. $X=Y$ represents for electrophiles. (a)

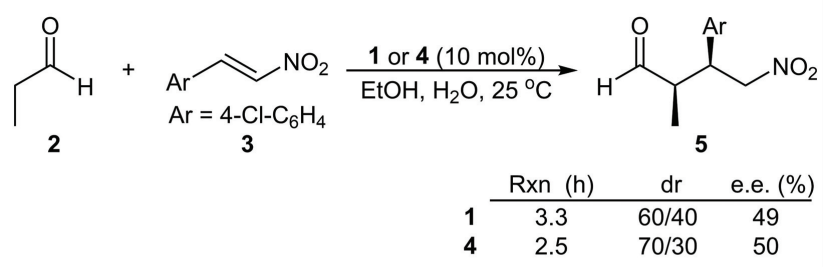

(b)<smiles>c1ccc(OCc2cn(C[C@@H]3CCCN3)nn2)cc1</smiles>

1 (c)

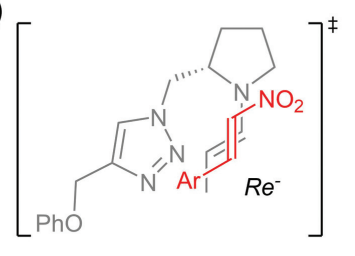

(d)

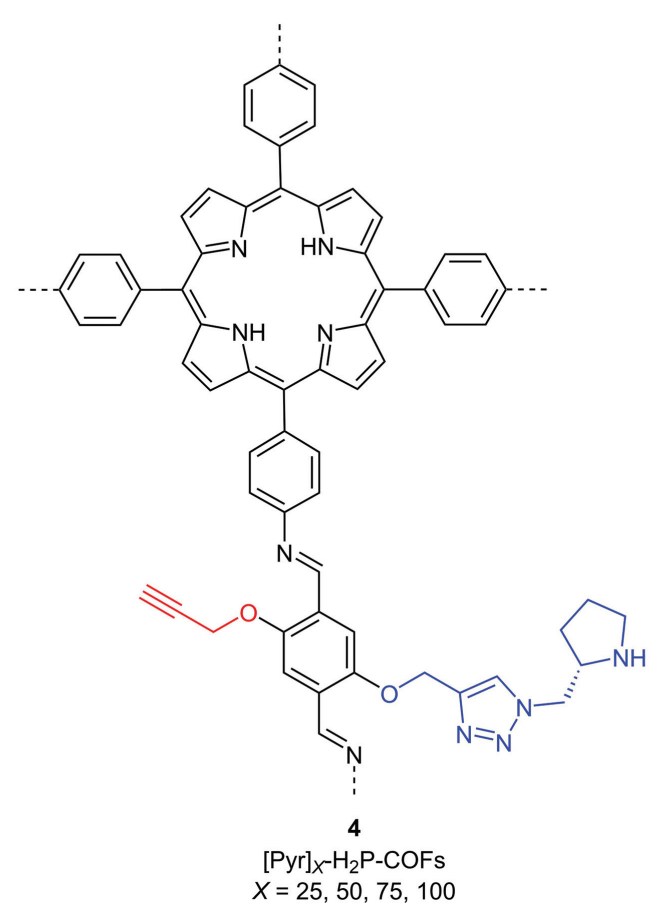

Figure 3 Michael addition catalyzed by chiral COF $[\mathrm{Pyr}]_{50}-\mathrm{H}_{2} \mathrm{P}-\mathrm{COF} 4$ and molecular catalyst $\mathbf{1}$, and proposed transition state of $\mathbf{1}$-catalyzed reaction.

transition state (Figure 3c). Jiang et al. re-explored this transformation by applying the click chemistry onto COF containing a terminal alkyne side chain. ${ }^{23}$ The identical catalytic pyrrolidine unit was homogeneously engineered onto the walls with typical $\mathrm{Cu}^{\mathrm{I}}$-promoted cyclization. The prepared COF 4, named in accordance with different amounts of pyrrolidine units ( $X=$ click proportion), was not dissolvable in water-ethanol solution, and was thus applied into the addition of aldehyde $\mathbf{2}$ and nitroalkene $\mathbf{3}$ in an aqueous solution (Figure $3 d$ ). It turned out the efficiency of organocatalytic COF was humbly modulated by the 
triazole amount, while $[\mathrm{Pyr}]_{50}-\mathrm{H}_{2} \mathrm{P}-\mathrm{COF}$ ( $50 \%$ of alkyne was modified) exhibited the best catalytic activity. The starting material was consumed in less than 3 hours and the desired product $\mathbf{5}$ was obtained in better diastereoselectivity ( $\mathrm{dr}$ 70/30) and retained enantioselectivity (e.e. 50\%). The same stereocontrol indicated that the activation model was identical to the molecular catalyst $\mathbf{1}$, while the acceleration was attributed to the one-dimensional channel with $\mathbf{4}$ that accommodated the reactants.

Compared with molecular catalysts, the biggest distinction of COF catalysts is that they are heterogeneous, which makes it easy to separate the catalyst from the reaction system, realizing the recycling of the catalyst, and can be used in flow chemistry. Thus, the prepared COF $\mathbf{4}$ was used at least 4 times with a slight decrease in activity. A flow reaction system based on the COF structure was also conducted. With that in hand, the enhanced activity, noble recyclability, and great capability to perform reactions under continuous flow conditions were all well demonstrated in this pioneering work.

In their continuous effort of designing new platforms for chiral organocatalysts, Jiang et al. later modified the existing COF 6 by introducing two methoxy groups, which increased the electron density of the central phenyl ring and thus improved the interlayer interactions and thus its stability (Figure 4). ${ }^{24} \mathrm{COF} \mathbf{6}$ and other analogues were reported to be the most stable COFs at that time, with almost no weigh loss $(<0.1 \mathrm{wt} \%)$ in organic solvents, aqueous $\mathrm{HCl}$, and $\mathrm{NaOH}$ solutions. This $\mathrm{COF} \mathbf{6}$, engineered with same organocatalytic triazole-based pyrrolidine sites on the wall, was able to promote the Michael addition of cyclohexanone 8 and nitroalkenes $\mathbf{3}$ in pure water, which was a nightmare for most organic reactions. ${ }^{43-45}$ Again, the molecular catalyst 1 showed a much lower activity, completing the addition in 22 hours, while $[(S) \text {-Py }]_{0.17}$-TPB-DMTP-COF $(17 \%$ sites modified) exhibited significant improvement. The reaction finished in 12 hours and afforded the desired adduct in comparable stereoselectivities ( $\mathrm{dr} 90 / 10$, e.e. 92\%). The enhanced catalytic ability was ascribed to the open channels that function as a confined nanopore reactor and accumulated the reactants from water. Not surprisingly, the grafted COF exhibited good reusability after five times with retained activity. Recently, Cui et al. prepared a similar pyrrolidinebased COF 7 but with a shorter linkage. ${ }^{28}$ Inferior diastereoselectivity and enantiostereoselectivity were observed, indicating that the length from the catalytic sites to the walls played an important factor in the transformation. However, no additional examples were provided to elucidate the relationships behind this.

In addition to pyrrolidine-based organotalysts for Michael additions, acyclic chiral secondary amines have also been developed for asymmetric Michael addition with stereochemical control comparable to or sometimes better than pyrrolidines. These molecules have many desirable

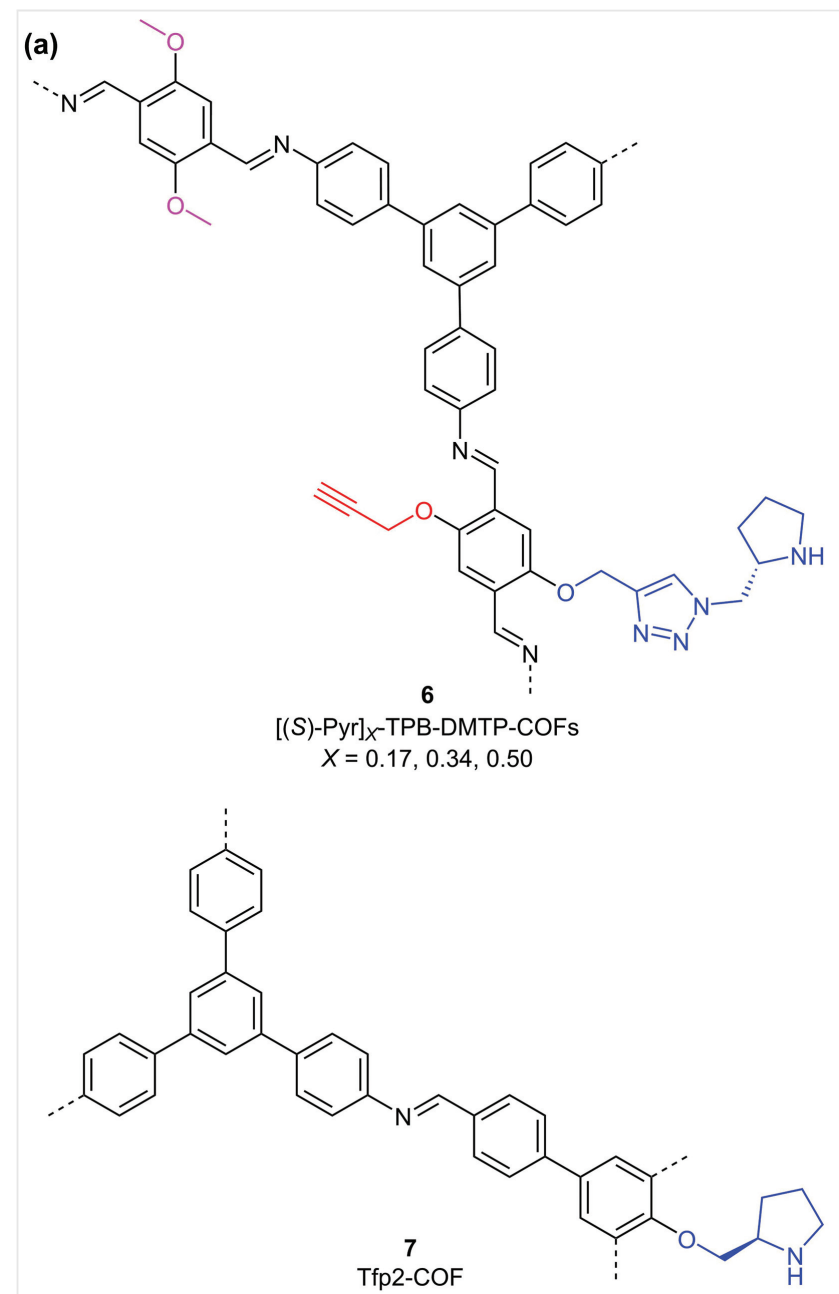

(b)

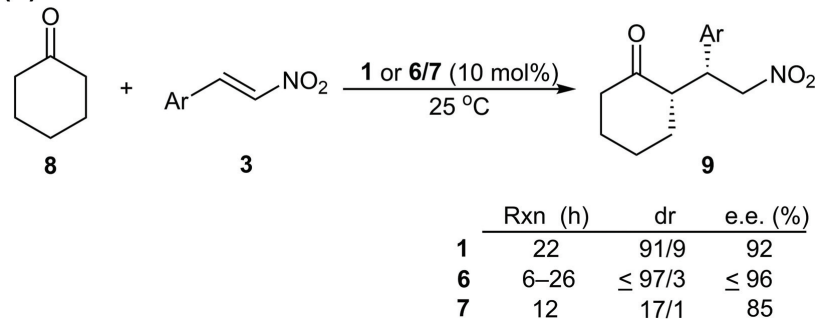

Figure 4 Michael reaction catalyzed by chiral COFs $[(S)-P y r]_{x}$ TPBDMTP-COFs 6 and Tfp2-COF 7.

features. They can be easily constructed from achiral pieces, thus producing a more diverse catalyst library than their cyclic analogues. However, the conformational free rotation may not be able to form a preferable transition state, making the asymmetric induction more challenging. Recently, Dong et al. investigated the acyclic amine-based $(R)$-DTP-COF 11 as a chiral catalyst into the Michael addition as mentioned 
(a)<smiles>C(#CC(Nc1ccccc1)c1ccccc1)c1ccccc1</smiles>

10 (b)

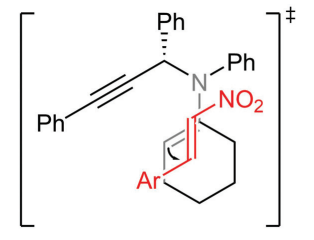

(c)

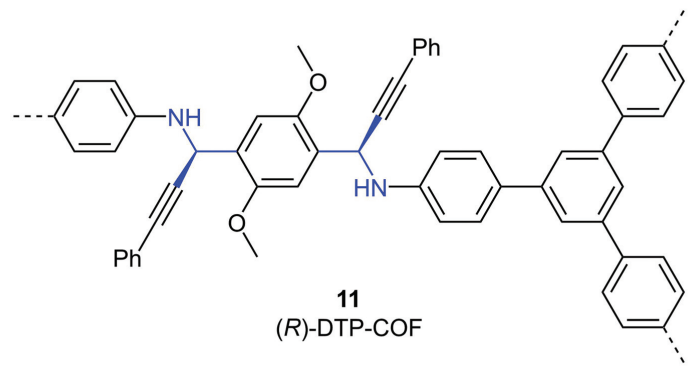

(d)

$$
\begin{aligned}
& \underbrace{}_{8}+\mathrm{NO}_{2} \frac{\begin{array}{c}
10 \text { or } 11 \\
\text { p-TsOH }(20 \mathrm{~mol} \%) \\
\mathrm{EtOH}, 25^{\circ} \mathrm{C}, 13 \mathrm{~h}
\end{array}}{3.3 \mathrm{~mol} \%, \mathrm{NH})} \\
& 8 \quad 3 \\
& 3 \\
& \begin{array}{rrrr} 
& \text { Yield (\%) } & \multicolumn{1}{c}{\text { dr }} & \text { e.e. (\%) } \\
\cline { 2 - 4 } & 21 & 55 / 45 & 74 \\
11 & \leq 99 & \leq 88 / 12 & \leq 99
\end{array}
\end{aligned}
$$

Figure 5 Michael reaction catalyzed by (R)-DTP-COF 11 and 10, and proposed transition state of this reaction.

above (Figure 5$) \cdot{ }^{27}$ They elegantly used a more sophisticated $\mathrm{C}-\mathrm{C}$ bond formation reaction - three-component coupling of an alkyne, an aldehyde, and an amine (A3-coupling) - into the construction of COF 11. ${ }^{46}$ This is by far the only example of constructing a chiral COF with enantioselective synthesis. The optical purity (e.e.) of the propargylamine linkage in the framework was as high as $93 \%$, as determined by optical rotation. This type of COFs is robust enough to resist acids, alkalis, and organic solvents. Surprisingly, it promoted the Michael addition of $\mathbf{8}$ with $\mathbf{3}$ in a heterogeneous manner with a much higher activity and stereocontrol. With $3.3 \mathrm{~mol}$ $\%$ of $\mathrm{NH}$ motif, the addition was furnished in excellent yield (97\%), moderate $\mathrm{dr}(72 / 28)$, and excellent e.e. (97\%). In contrast, the homogeneous motif $\mathbf{1 0}$ provided a much lower yield (21\%) and inferior $\mathrm{dr}$ (55/45) and e.e. (74\%). In addition to the better activity and chirality control, COF 11 possessed an interesting substrate size discrimination. Larger substrates like $\beta$-tetralone with 9-(2-nitrovinyl)anthracene did not afford the desired adduct, indicating an enzyme-like preference.

The Aldol reaction is one of the classic carbonyl condensation reactions involving aldehydes and/or ketones to yield $\alpha$-hydroxy carbonyl compounds. ${ }^{47}$ Again, secondary amines would react with appropriate ketones to form

transient enamines, which then attack enantioselectively with suitable electrophiles, such as aldehydes. ${ }^{48}$ Over the past two decades, a number of highly enantioselective variants of the Aldol reactions under the catalysis of secondary and primary amines have been reported. However, no COF-based organocatalyst has been developed until Wang et al. for the first time grafted the pyrrolidine ring onto a COF via the condensation of chiral aryl diamine with terphenyl monomers. ${ }^{25}$ The robust $\beta$-ketoenamine linkage was stable under acidic conditions, and yet possessed hexagonal pores, high crystallinity, and porosity. ${ }^{11}$ The pyrrolidine sites were evenly distributed in the open channels and thus promoted the Aldol reaction of acetone $\mathbf{1 2}$ with aryl aldehydes $\mathbf{1 3}$ with comparable enantioselectivity (88\%). However, a much longer reaction time was required (18-96 h) (Figure 6).

(a)<smiles>CC(=O)[14CH2]C=O</smiles>

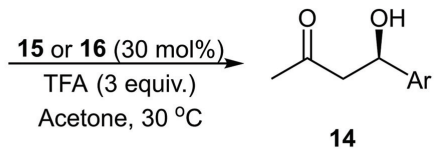

(b)<smiles></smiles>

(c)

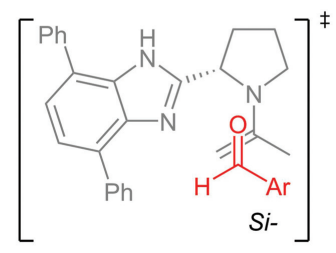

(d)<smiles>CN=Cc1cc(C=N)cc(C=Nc2ccc(-c3ccc(-c4ccc(I)cc4)c4[nH]c([C@@H]5CCCN5)nc34)cc2)c1</smiles><smiles>CNC=C1C(=O)C(=CNC)C(=O)C(=CNc2ccc(-c3ccc(-c4ccc(C)cc4)c4[nH]c([C@@H]5CCCN5)nc34)cc2)C1=O</smiles>

Figure 6 Aldol reaction catalyzed by LZU-72/73 (16a/b), and proposed transition state of this reaction. 
Recently, the same group reported their efforts in preparing more diverse chiral COFs via post-modification of the monomers with chiral amines. ${ }^{26}$ Various aminoalcohols, diamines, and cyclic amines were prepared in this way and hence chiral COFs. The simple introduction of Bronsted acid and Lewis base sites enabled a dualfunctionalization of two substrates, just like those reported bifunctional organocatalysts. ${ }^{49,50}$ In addition to those C-C bond formations, the development of creating $\mathrm{C}-\mathrm{N}$ bonds is also at the research forefront in synthetic community. ${ }^{51}$ While many classical transformations have been established, direct addition to azodicarboxylates is one of the most useful protocols. ${ }^{52}$ To this end, Wang et al. applied their bifunctional chiral COFs into the amination of ethyl 2oxocyclopentane-1-carboxylate 17 with di-tert-butyl diazene-1,2-dicarboxylate 18. It turned out that the cyclohexane-1,2-diamine-based TAH-CCOF2 21 afforded the product 19 in identical chirality as to the molecular motif $\mathbf{2 0}$, indicating that they should undergo the same transition state. As shown in Figure 7, the $N, N$-dimethyl amine abstracted one hydrogen from the dicarbonyl to generate the enolate which was stabilized by the $1 H$-benzo[d] imidazol-2-amine motif via two distinct hydrogen bondings. ${ }^{53}$ The orientation was induced by both the cyclohexane structure and the open channel. It was proposed by the authors that grafting onto the steric bulk COF walls may weaken the intermolecular assembly of catalytic sites via hydrogen bonding and thus benefit the steric control and catalytic activity. Besides, the heterogeneous COF can be easily recovered and reused for seven times without any obvious loss of effectiveness, demonstrating its potential application in flow systems.

In addition to the examples introduced above, Cui et al. have greatly extended the application range of the pyrrolidine catalyst from Aldol reaction to asymmetric aminooxylation reaction and the Diels-Alder cyclization. ${ }^{29}$ They adopted the dimethoxyl-substituted strategy as mentioned before ${ }^{24}$ and further introduced four different L-proline/L-imidazolidine motifs onto the walls. Through a sophisticatedly designed protection-deprotection strategy, they were able to generate four chiral COFs 22a-d (Figure 8a). The introduction of different chiral segments led to a systemic change in the crystallinity and thus should have diverse catalysis abilities. Indeed, in spite of traditional Aldol reactions as discussed before, the authors explored the application of chiral COFs and their homogeneous molecules 23a-c (Figure 8b) in the asymmetric aminooxylation of aldehydes (Figure 8c) and Diels-Alder cyclization (Figure 8d). Chiral COF 22b showed comparable activity with its homogeneous 23a in the $\alpha$-aminooxylation of aldehydes 24 with nitrosobenzene 25, exhibiting an identical $N$-selectivity. However, they showed a significant discrimination of endo/exo selectivity in the Diels-Alder reaction of aldehydes $\mathbf{2 7}$ and cyclopentadiene $\mathbf{2 8}$. In most (a)

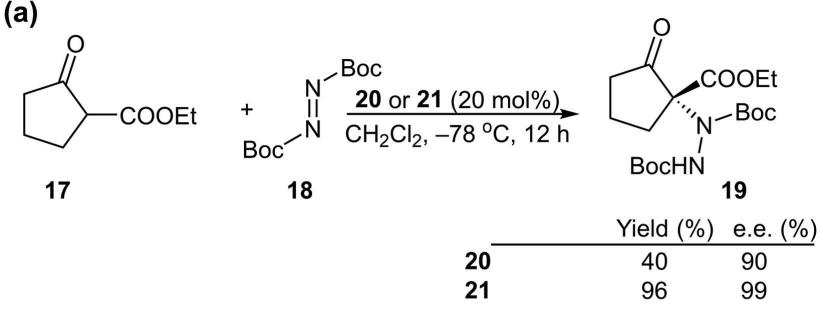

(b)

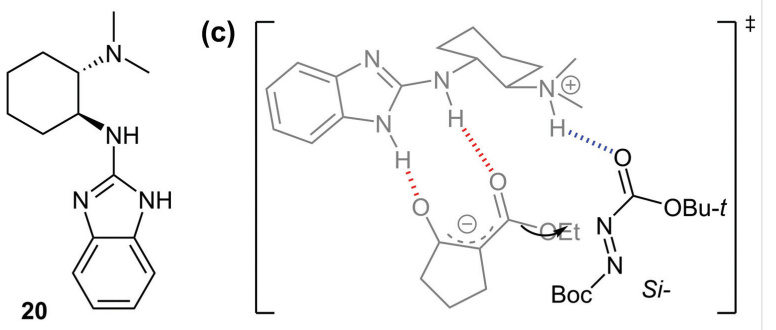

(d)

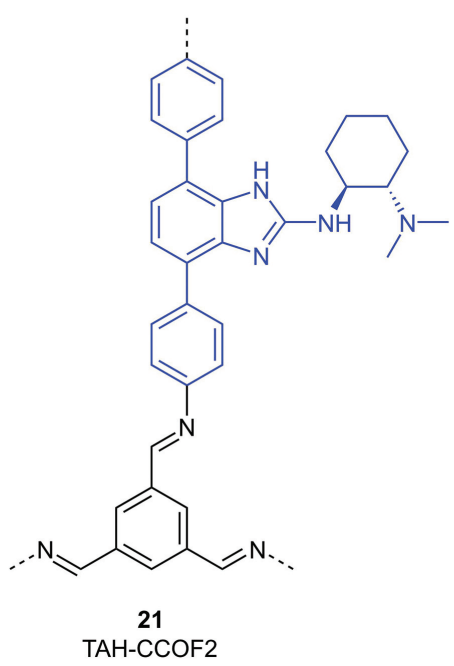

Figure 7 Asymmetric C-N bond formation catalyzed by TAH-CCOF 2 (21) and the proposed transition state.

cases, chiral COF afforded the product in 4-7 times greater diastereoselectivities and in comparable enantioselectivities, indicating that the stereocontrol occurred within the catalytic sites, just like with molecular catalysts. However, the open channel may have afforded additional secondary interactions, directing either the generated iminium cation or the diene to a more energy-preferable transition state. It is also worth noting that simply changing the parasubstituent in the cinnamaldehyde led to a dramatic endo/exo selectivity switch. No reasonable explanation was given at this stage, but it seems that the chiral COF accelerated the cyclization without distinction to the substituent. The authors also compared their synthetic COFs with amorphous catalysts, again proving that the crystallinity and porosity of COFs play an important role in asymmetric transformations. Very recently, the same 
(a)

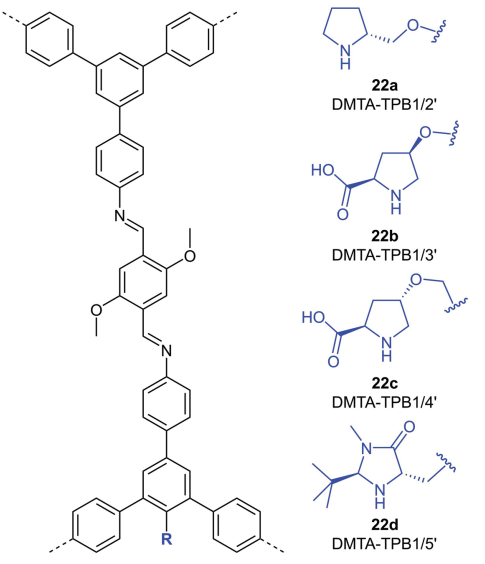

(b)

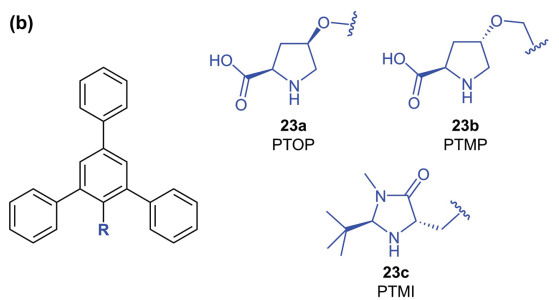

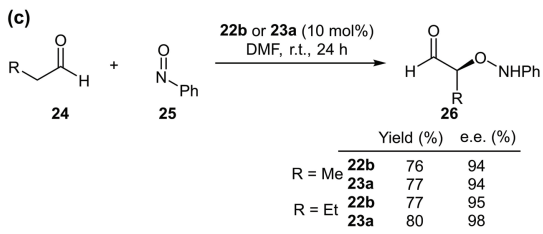

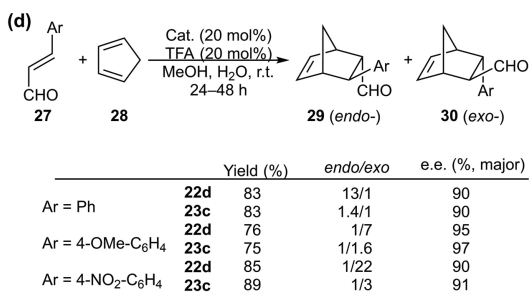

(e)

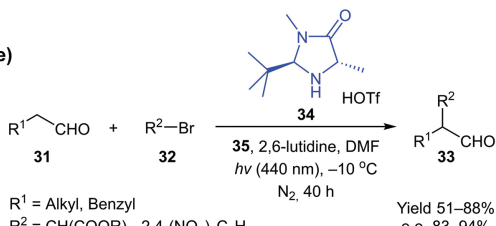

$\begin{array}{ll}\mathrm{R}^{1} & =\text { Alkyl, Benzyl } \\ \mathrm{R}^{2}=\mathrm{CH}(\mathrm{COOR})_{2}, 2,-\left(\mathrm{NO}_{2}\right)_{2} \mathrm{C}_{6} \mathrm{H}_{4} & \text { Yield } 51-88 \%\end{array}$

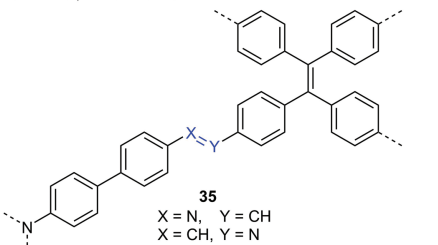

Figure 8 Asymmetric aminooxylation reaction and Diels-Alder cyclization catalyzed by chiral COFs 22 and their homogeneous analogs 23 and $\alpha$-alkylation of aldehydes by incubating COF 35 with Macmillan catalyst 34 . authors rationally synthesized two interpenetrated threedimensional COFs 35 containing an infrequent $(3,4)$ connected ffc topology. ${ }^{54}$ The inherent triarylamine structure was an efficient photocatalyst for cross-dehydrogenative coupling transformations. Besides, these COFs were able to encapsulate aliphatic aldehydes $\mathbf{3 1}$, activated bromides 32, and the Macmillan catalyst $\mathbf{3 4}$ in the asymmetric $\alpha$-alkylation (Figure 8e). Good yields and enantioselectivities of the products $\mathbf{3 3}$ were obtained in most cases, showing a similar activation model in a homogeneous situation. These COFs lost their crystallinity after the reaction, but can be restored by heating, indicating a possible reusable potential.

Cui et al. reported the only chiral COF containing tertiary amine functionality that has been utilized in asymmetric organocatalysis. ${ }^{28}$ The authors smartly grafted the chiral bicycle imidazole nucleophilic catalyst 6,7-dihydro-5Hpyrrolo[1,2-a]imidazole (abbreviated as DHIP) ${ }^{55}$ and prepared the chiral COF TPB2-COF 36, which was later employed in the same asymmetric Steglich rearrangement of (S)-oxazol-5-yl carbonates $\mathbf{3 7}$ to generate the chiral 5oxo-4,5-dihydrooxazole-4-carboxylates 38 (Figure 9). Comparable activity was observed, however, in less satisfactory enantioselectivities (up to $84 \%$ ). The reaction was accomplished in as long as 4 days, presumably due to the catalytic site efficiency as well as the steric hindrance within the<smiles>Cc1ccc(-c2cc(-c3ccc(C)cc3)cc(-c3ccc(/N=C/c4ccc(-c5cc(C)c(COC6CCn7ccnc76)c(F)c5)cc4)cc3)c2)cc1</smiles>

(b)

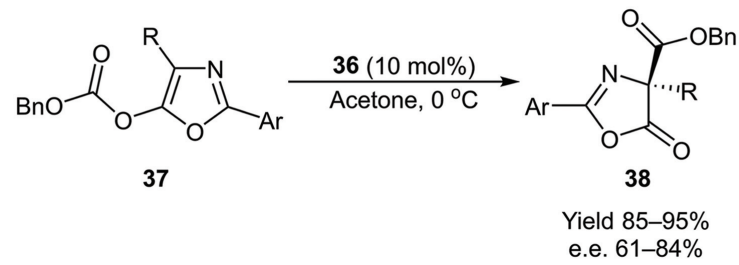

Figure 9 Asymmetric Steglich rearrangement catalyzed by TPB3-COF 36. 
nanopore walls. Nonetheless, this example has shed light on the potential application of chiral COFs in more transformations other than carbonyl activation.

\section{Conclusions and Outlook}

Chiral COFs, or more precisely, chiral covalent organocatalytic frameworks, are a unique class in polymer materials. They possess chiral side chains or asymmetric backbones, and yet maintain the highly organized frameworks, which distinguish themselves from traditional enantiopure small organic molecules or chiral polymers. However, the design and construction of those porous materials is still in the infancy. In this Short Review, we have briefly summarized a few examples that have been reported about the successful construction of chiral COFs and their application in asymmetric catalysis. Although these works have paved the way for further utilization of these materials, some significant limitations still exist. First, the majority of these materials were restricted in grafting chiral motifs onto the achiral COFs, which may greatly influence the structure or even stability of the existing framework. Only a few of these research studies tried to modify the structure of COFs to achieve superior functions. It is reasonable that current research predominately neglected this structure-activity relationship study. However, the structural illumination of such heterogenized catalysts is a critical stage to comprehend their catalytic performance, no matter they exhibited better or worse activities compared with the corresponding catalytic motif. A lack of techniques that can precisely describe the orientation/distribution of each chiral site may be one of the reasons here. Second, the open channel or nanopore confinement has not been fully appreciated in most cases. In another word, no significant kinetic acceleration and/or stereocontrol was observed in most of those examples. ${ }^{23-26,28,29}$ Considering the fact that enzymecatalyzed reactions are momentously accelerated by the substrate binding in a confined space that helps it to catalyze the process with numerous secondary interactions within amino acid residues around the space, it is promising that chemists could also design various well-defined hydrophobic cavities within the COF walls to mimic the enzyme's active site. Of course, that would require building blocks that are more sophisticated during the COF construction to maintain the organized structure. Third, the current activation models are predominately limited in the enamine and iminium catalysis models with secondary amines. Other manners like hydrogen-bonding catalysis, SOMO (singly occupied molecular orbital) catalysis, and counterion catalysis have not been realized yet. Two reasons may be responsible for this. One of the difficulties of introducing well-established organocatalysts onto existing COFs may be due to the limited post-modification strategy.
Click chemistry is one of the most utilized methods, but it may hamper the micro- and steric environment around the catalytic site. Suzuki coupling would be a promising pathway, but the strong alkali reaction condition and the poor functionality tolerance may limit the choice of organocatalyst. Another problem may be due to the usually large size of organic molecules currently utilized in asymmetric synthesis. Post-introduction of these motifs, either within the walls or outside the COF, they may have a significant impact on the stability and crystalline form of $\mathrm{COF}$, and hence led to unpredictable influence on their catalytic abilities. Nonetheless, we envisioned that the potentials of chiral COFs are far beyond these examples mentioned in this Review. Given the asymmetric environment of the highly organized framework, it may serve as an efficient accelerator for some reactions. Those materials, with structure mimicking natural enzymes, may serve as heterogeneous catalysts in large-scale synthesis, or even in industrial manufacturing processes. With more and more synthetic chemists and materials scientist devoting to this area, chiral COFs with more interesting proprieties will be prepared and their further application will be booming.

\section{Funding Information}

This work was supported by the National Key Research and Development Program of China (2016YFA0602900) and Chinese Academy of Sciences.

\section{References}

(1) Wagnière, G. H. On Chirality and the Universal Asymmetry: Reflections on Image and Mirror Image. John Wiley \& Sons: Weinheim, 2007.

(2) Cintas, P. Angew. Chem. Int. Ed. 2007, 46, 4016.

(3) Calcaterra, A.; D’Acquarica, I. J. Pharm. Biomed. Anal. 2018, 147, 323.

(4) Trost, B. M. Proc. Natl. Acad. Sci. U.S.A. 2004, 101, 5348.

(5) Carreira, E. M.; Jacobsen, E. N.; Pfaltz, A.; Yamamoto, H. Comprehensive Asymmetric Catalysis. Springer: Berlin, 1999.

(6) Ojima, I.Ed. Catalytic Asymmetric Synthesis. John Wiley \& Sons: Weinheim, 2010.

(7) Itsuno, I. S.; Parvez, M. M.; Haraguchi, N. Polym. Chem. 2011, 2, 1942.

(8) Côté, A. P.; Benin, A. I.; Ockwig, N. W.; O’Keeffe, M.; Matzger, A. J.; Yaghi, O. M. Science 2005, 310, 1166.

(9) Ding, S.-Y.; Gao, J.; Wang, Q.; Zhang, Y.; Song, W.-G.; Su, C.-Y.; Wang, W. J. Am. Chem. Soc. 2011, 133, 19816.

(10) Furukawa, H.; Yaghi, O. M. J. Am. Chem. Soc. 2009, 131, 8875.

(11) Kandambeth, S.; Mallick, A.; Lukose, B.; Mane, M. V.; Heine, T.; Banerjee, R. J. Am. Chem. Soc. 2012, 134, 19524.

(12) Uribe-Romo, F. J.; Hunt, J. R.; Furukawa, H.; Klöck, C.; O’Keeffe, M.; Yaghi, O. M. J. Am. Chem. Soc. 2009, 131, 4570.

(13) Ding, S.-Y.; Wang, W. Chem. Soc. Rev. 2013, 42, 548.

(14) Feng, X.; Ding, X.; Jiang, D. Chem. Soc. Rev. 2012, 41, 6010. 


\section{Organic Materials S.-C. Yu et al.}

(15) Geng, K.; He, T.; Liu, R.; Dalapati, S.; Tan, K. T.; Li, Z.; Tao, S.; Gong, Y.; Jiang, Q.; Jiang, D. Chem. Rev. 2020, 120, 8814.

(16) Huang, N.; Wang, P.; Jiang, D. Nat. Rev. Mater. 2016, 1, 16068.

(17) Kandambeth, S.; Dey, K.; Banerjee, R. J. Am. Chem. Soc. 2019, 141, 1807.

(18) Guan, Q.; Zhou, L.-L.; Li, W.-Y.; Li, Y.-A.; Dong, Y.-B. Chem. Eur. J. 2020, 26, 5583.

(19) Haug, W. K.; Moscarello, E. M.; Wolfson, E. R.; McGrier, P. L. Chem. Soc. Rev. 2020, 49, 839.

(20) Liu, J.; Wang, N.; Ma, L. Chem. Asian J. 2020, 15, 338.

(21) Sun, T.; Xie, J.; Guo, W.; Li, D.-S.; Zhang, Q. Adv. Energy Mater. 2020, 10, 1904199.

(22) Wang, Z.; Zhang, S.; Chen, Y.; Zhang, Z.; Ma, S. Chem. Soc. Rev. 2020, 49, 708.

(23) Xu, H.; Chen, X.; Gao, J.; Lin, J.; Addicoat, M.; Irle, S.; Jiang, D. Chem. Commun. 2014, 50, 1292.

(24) Xu, H.; Gao, J.; Jiang, D. Nat. Chem. 2015, 7, 905.

(25) Xu, H.-S.; Ding, S.-Y.; An, W.-K.; Wu, H.; Wang, W. J. Am. Chem. Soc. 2016, 138, 11489.

(26) Wang, L.-K.; Zhou, J.-J.; Lan, Y.-B.; Ding, S.-Y.; Yu, W.; Wang, W. Angew. Chem. Int. Ed. 2019, 58, 9443.

(27) Wang, J.-C.; Kan, X.; Shang, J.-Y.; Qiao, H.; Dong, Y.-B.J. Am. Chem. Soc. 2020, 142, 16915.

(28) Zhang, J.; Han, X.; Wu, X.; Liu, Y.; Cui, Y. ACS Sustainable Chem. Eng. 2019, 7, 5065.

(29) Zhang, J.; Han, X.; Wu, X.; Liu, Y.; Cui, Y. J. Am. Chem. Soc. 2017, $139,8277$.

(30) Dong, J.; Han, X.; Liu, Y.; Li, H.; Cui, Y. Angew. Chem. Int. Ed. 2020, 59, 13722.

(31) Han, X.; Yuan, C.; Hou, B.; Liu, L.; Li, H.; Liu, Y.; Cui, Y. Chem. Soc. Rev. 2020, 49, 6248.

(32) Ma, H. C.; Zou, J.; Li, X. T.; Chen, G. J.; Dong, Y. B. Chem. Eur. J. 2020, 26, 13754.

(33) Wang, X.; Han, X.; Zhang, J.; Wu, X.; Liu, Y.; Cui, Y. J. Am. Chem. Soc. 2016, 138, 12332.
(34) Han, X.; Zhang, J.; Huang, J.; Wu, X.; Yuan, D.; Liu, Y.; Cui, Y. Nat. Commun. 2018, 9, 1294.

(35) Mukherjee, S.; Yang, J. W.; Hoffmann, S.; List, B. Chem. Rev. 2007, 107, 5471.

(36) Pihko, P. M.; Majander, I.; Erkkilä, A. Top. Curr. Chem. 2010, 291, 29.

(37) Dalko, P. I.; Moisan, L. Angew. Chem. Int. Ed. 2004, 43, 5138.

(38) Notz, W.; Tanaka, F.; Barbas, C. F. III Acc. Chem. Res. 2004, 37, 580.

(39) List, B. Acc. Chem. Res. 2004, 37, 548.

(40) List, B. Tetrahedron 2002, 58, 5573.

(41) Berner, O. M.; Tedeschi, L.; Enders, D. Eur. J. Org. Chem. 2002, 2002,1877

(42) Luo, S.; Xu, H.; Mi, X.; Li, J.; Zheng, X.; Cheng, J. P. J. Org. Chem. 2006, 71, 9244.

(43) Kitanosono, T.; Kobayashi, S. Chem. Eur. J. 2020, 26, 9408.

(44) Kobayashi, S. Pure Appl. Chem. 2007, 79, 235.

(45) van Der Helm, M. P.; Klemm, B.; Eelkema, R. Nat. Rev. Mater. 2019, 3, 491.

(46) Wei, C.; Li, Z.; Li, C.-J. Synlett 2004, 1472.

(47) Trost, B. M.; Brindle, C. S. Chem. Soc. Rev. 2010, 39, 1600.

(48) Gotoh, H.; Hayashi, Y. C-C Bond Formation: Aldol Reaction with Non-Proline Derivatives. In: Comprehensive Chirality. Carreira, E. M.; Yamamoto, H. Elsevier Ltd.: Amsterdam, 2012, 125-156.

(49) Okino, T.; Hoashi, Y.; Furukawa, T.; Xu, X.; Takemoto, Y. J. Am. Chem. Soc. 2005, 127, 119.

(50) Okino, T.; Hoashi, Y.; Takemoto, Y. J. Am. Chem. Soc. 2003, 125, 12672.

(51) Bariwal, J.; Van der Eycken, E. Chem. Soc. Rev. 2013, 42, 9283.

(52) Usman, M.; Zhang, X. W.; Wu, D.; Guan, Z. H.; Liu, W. B. Org. Chem. Front. 2019, 6, 1905.

(53) Trillo, P.; Gómez-Martínez, M.; Alonso, D. A.; Baeza, A. Synlett 2015, 95.

(54) Kang, X.; Wu, X.; Han, X.; Yuan, C.; Liu, Y.; Cui, Y. Chem. Sci. 2020, $11,1494$.

(55) Zhang, Z.; Xie, F.; Jia, J.; Zhang, W. J. Am. Chem. Soc. 2010, 132, 15939 . 